\title{
Apparent multifractality of self-similar Lévy processes
}

\author{
Marco Zamparo \\ Dipartimento Scienza Applicata e Tecnologia and CNISM, Politecnico di Torino, \\ Corso Duca degli Abruzzi 24, I-10129 Torino, Italy \\ E-mail: marco.zamparo@polito.it
}

\begin{abstract}
Scaling properties of time series are usually studied in terms of the scaling laws of empirical moments, which are the time average estimates of moments of the dynamic variable. Nonlinearities in the scaling function of empirical moments are generally regarded as a sign of multifractality in the data. We show that, except for the Brownian motion, this method fails to disclose the correct monofractal nature of self-similar Lévy processes. We prove that for this class of processes it produces apparent multifractality characterised by a piecewise-linear scaling function with two different regimes, which match at the stability index of the considered process. This result is motivated by previous numerical evidence. It is obtained by introducing an appropriate stochastic normalisation which is able to cure empirical moments, without hiding their dependence on time, when moments they aim at estimating do not exist.
\end{abstract}

Keywords: Lévy processes, empirical moments, scaling laws 


\section{Introduction}

Many variables in natural and economic sciences appear to have some scale invariance properties. Since the beginning of fractal geometry with the work of Mandelbrot in 1960's, statistical self-similarity and scaling laws have been discovered in turbulence [1], ecology [2], hydrology [3], network data traffic [4], and finance [5] among other fields. In the specific context of time series, time-scale invariance for the moments of the dynamic

variable is generally observed [5]. The scaling law of different moments is completely described by a single exponent for monofractal dynamics, such as those corresponding to self-similar processes, whereas a continuous spectrum of exponents is needed for multifractal evolutions. The monofractal and the multifractal scaling properties are commonly regarded as fundamentally different, the former arising from additive and the latter from multiplicative processes [6,7].

Although multifractality is very attractive, there is a certain controversy over its emergence and nature, especially in finance. While many authors have provided evidence of multifractality in stock markets using different methods on the one hand [8 13], several papers report on the other hand that no sign of multifractal scaling laws is found in financial time series [14 16. In addition to this, spurious multifractal effects have been documented for different model types that in principle do not account for such a form of scaling properties [17 25]. This casts doubt on whether the observed multifractality always corresponds to a genuine multifractal process.

Multifractality in time series is detected by inspecting the power law in time of empirical moments, which are the time average estimates of moments, and evaluating their scaling function for various orders [7]. A linear scaling function corresponds to a monofractal process, whereas a nonlinear scaling function should denote the occurrence of multifractality. The basic assumption underlying this protocol is that moments exist. If this assumption is violated, then unforeseen and misleading results may be obtained, such as in the case of self-similar Lévy processes different from the Brownian motion. In fact, for these processes the scaling function of empirical moments results to be piecewise linear with two different regimes when the range of existing moments is supposed to be not known in advance, as argued in [8] and [17] on the basis of numerical simulations. A similar accident occurs for the fractional Lévy motion [20]. Piecewise linearity of the scaling function would suggest a multifractal model even though self-similar Lévy processes and the fractional Lévy motion are exactly monofractal processes. The nonlinearity of the scaling function is entirely caused by the non-existence of some moments, which for both model classes is due to the presence of fat tails in the distribution of the dynamic variable. This suggests that empirical facts considered as hallmarks of multifractal systems may be reproduced by plain fat-tailed processes, so that an apparent multifractality may be consistent with the latter. This circumstance deserves attention in finance, where variables are widely believed to possess fat tails [26] and where scaling functions approximately exhibiting piecewise linearity with two different regimes have already been found in several daily 
exchange rates [27]. Interestingly, scaling functions which share this same shape have also been found in the context of the natural sciences, such as solar wind data [28], diffusion in living cells [29], and transport phenomena in optical lattices [30].

Discerning real from apparent multifractality demands a thorough study of the estimators of moments with the purpose of isolating the mechanisms that may lead to spurious effects. The probability distribution of these estimators has been obtained for self-similar Lévy processes and the fractional Lévy motion in the limit of long time series, where scaling laws in time of the distribution emerge [20]. Within a special protocol, where the time horizon to which scaling laws should refer is allowed to grow as the sample size increases, similar results have been achieved for processes with more general independent and identically distributed fat-tailed increments [21]. The latter work has very recently been extended to models with stationary and weakly dependent increments [25]. These studies are however only partially informative as one must keep an eye on the fact that a deep insight into apparent multifractality of time series requires to understand whether or not and how the single empirical moments can exhibit multifractal scaling laws when the underlying process is not a multifractal process, as observed in [8] and [17]. The scaling properties of the single empirical moments are not directly related to those of their distribution, so that invoking the latter does not help to unravel the knot. To this aim, stronger results are needed and should be reached by somehow improving the weak convergence in distribution to the almost sure convergence, which is the only one that can explain what occurs in one single experiment.

In this paper we reconsider the problem of apparent multifractality of self-similar Lévy processes. We show that, except for the Brownian motion, empirical moments systematically exhibit a multifractal scaling law for almost all the sample paths of the underlying self-similar Lévy process, mistakenly leading to consider it a multifractal model. While non-degenerate distributions and their scaling properties can be obtained by resorting to norming constants only, the strategy we use to highlight the scaling laws of the single empirical moments when moments do not exist is employing a stochastic normalisation. A stochastic normalisation is made necessary by an important result due to Feller on sums of independent and identically distributed random variables that are not integrable. This strategy allows us to completely explain the numerical findings of [8] and [17] by demonstrating that empirical moments of any order acquire their own deterministic scaling laws with a well-defined scaling function in the limit of long time series. The scaling function is piecewise-linear with two different regimes matching at the stability index of the underlying process.

The paper is organised as follows. In Section 2 we recall some fundamental notions that are concerned with multifractal processes and the statistical methods for estimating their scaling function. In Section 3 we introduce self-similar Lévy processes and we review previously known facts about their apparent multifractality. We supplement the literature showing impossibility of disclosing multifractal scaling laws of empirical moments by resorting to norming constants only. Then we discuss a natural stochastic candidate for normalisation purposes and we state our main result on the apparent 
multifractality of self-similar Lévy processes. The proof of this result is outlined afterwards, postponing the most technical details that are needed in the appendices in order not to interrupt the flow of the presentation. Conclusions and prospects for future research are finally reported in Section 4 .

\section{Scaling properties of stochastic processes and empirical analysis}

Let on the probability space $(\Omega, \mathcal{F}, \mathbb{P}) \dagger$ be given a continuous-time real-valued stochastic process $\left\{X_{t}\right\}_{t \geq 0}$ satisfying $X_{0}=0$ and having stationary increments. We recall that the increment of $\left\{X_{t}\right\}_{t \geq 0}$ at time $t$ and scale $s$, or over the time window from $t$ to $t+s$, is the variable $\Delta_{t}^{s} X:=X_{t+s}-X_{t}$ and that the process possesses stationary increments if $\Delta_{t}^{s} X \stackrel{d}{=} \Delta_{0}^{s} X$ for all $t>0$ and $s>0$, where equality is in distribution. Scale-invariance properties of $\left\{X_{t}\right\}_{t \geq 0}$ can be defined by specifying the scaling rules of moments [6, 7]. The process is said to be multifractal if there exist some real numbers $T>0$ and $b>0$ and some functions $\mu$ and $\nu$ with domain $[0, b)$ such that the scaling law

$$
\mathbb{E}\left[\left|X_{t}\right|^{q}\right]=\mu(q) \cdot t^{\nu(q)}
$$

holds for all $t \leq T$ and non-negative $q<b$. The quantity $\mathbb{E}\left[\left|X_{t}\right|^{q}\right]$ is the moment of the process at time $t$ and order $q$. The function $\nu$ is the scaling function and necessarily $\nu(0)=0$ and $\nu$ is concave [6,7]. The simplest multifractal processes are characterised by a linear scaling function and are also termed monofractal. Self-similar processes constitute an example of monofractal processes, whereas an example of multifractal processes showing non linearities in the scaling function is represented by the socalled log-infinitely divisible multifractal processes [31,32]. We recall that the process $\left\{X_{t}\right\}_{t \geq 0}$ is said to be self-similar if there exists a scaling exponent $H>0$ such that $X_{a t} \stackrel{d}{=} a^{H} X_{t}$ for all $a>0$ and $t>0$. Self-similar processes verify (1) for all $t$ with $b=\sup \left\{q \geq 0: \mathbb{E}\left[\left|X_{1}\right|^{q}\right]<\infty\right\}, \mu(q)=\mathbb{E}\left[\left|X_{1}\right|^{q}\right]$, and $\nu(q)=H q$.

Empirical scaling analysis aims at assessing scaling properties of the process $\left\{X_{t}\right\}_{t \geq 0}$ on the basis of time series data and requires estimation of the moment $\mathbb{E}\left[\left|X_{\tau}\right|^{q}\right]$ for several times $\tau$ and orders $q$. The value $\nu(q)$ of the scaling function at $q$ is evaluated by linear regression of logarithms of estimated moments on $\ln \tau$ [7]. In the typical experiment, $N+1$ measurements of the process are recorded at the equally spaced time instants $0=\tau_{0}<\tau_{1}<\cdots<\tau_{N}$ and the times $\tau$ identifying moments to be estimated are multiples of the lag between consecutive measurements. We choose unit of time in such a way that $\tau_{1}=1$, so that $\tau_{n}=n$ for all $n$ and times $\tau$ are integers. Within this framework, the estimator ${ }^{q} M_{N}^{\tau}$ of the moment $\mathbb{E}\left[\left|X_{\tau}\right|^{q}\right]$ is defined as the sample average of the increments of $\left\{X_{t}\right\}_{t \geq 0}$ over $N / \tau$ non-overlapping, consecutive time windows of

$\ddagger$ As usual, $\Omega$ denotes the sample space, $\mathcal{F}$ is a $\sigma$-algebra of events on $\Omega$, and $\mathbb{P}$ is a probability measure on $\mathcal{F}$. A property holds almost surely (a.s. for short) if it holds for all the samples $\omega$ belonging to an event $E \in \mathcal{F}$ with $\mathbb{P}[E]=1$. 
size $\tau$ :

$$
{ }^{q} M_{N}^{\tau}:=\frac{1}{N / \tau} \sum_{n=0}^{N / \tau-1}\left|\Delta_{\tau n}^{\tau} X\right|^{q}=\frac{1}{N / \tau} \sum_{n=1}^{N / \tau}\left|X_{n \tau}-X_{(n-1) \tau}\right|^{q} .
$$

The realisation ${ }^{q} M_{N}^{\tau}(\omega)$ of the random variable ${ }^{q} M_{N}^{\tau}$ corresponding to the available data $0=X_{0}(\omega), X_{1}(\omega), \ldots, X_{N}(\omega)$ is called empirical moment and precisely constitutes the estimate of $\mathbb{E}\left[\left|X_{\tau}\right|^{q}\right]$. With the purpose of estimating the moment $\mathbb{E}\left[\left|X_{\tau}\right|^{q}\right]$ for all $\tau$ up to a certain given integer temporal horizon $\mathcal{T}$, the number of measurements $N$ is assumed to be a multiple of the least common multiple $\operatorname{lcm}(\mathcal{T})$ of the first $\mathcal{T}$ integers. This way, the number $N / \tau$ of consecutive increments that appears in (2) is an integer for all $\tau \leq \mathcal{T}$. In practical situations, $N / \mathcal{T}$ has to be large in order to collect enough statistics.

The hypothesis of stationary increments yields that $\mathbb{E}\left[{ }^{q} M_{N}^{\tau}\right]=\mathbb{E}\left[\left|X_{\tau}\right|^{q}\right]$ for all those times $\tau \leq \mathcal{T}$ and orders $q$ that satisfy $\mathbb{E}\left[\left|X_{\tau}\right|^{q}\right]<\infty$, so that ${ }^{q} M_{N}^{\tau}$ is an unbiased estimator. We notice that $\mathbb{E}\left[\left|X_{\tau}\right|^{q}\right]<\infty$ for all integers $\tau$ if and only if $\mathbb{E}\left[\left|X_{1}\right|^{q}\right]<\infty$. The estimation procedure is said to be consistent if for all $q$ such that $\mathbb{E}\left[\left|X_{1}\right|^{q}\right]<\infty$

$$
\mathbb{P}\left[\bigcap_{\tau=1}^{\mathcal{T}}\left\{\omega \in \Omega: \lim _{k \uparrow \infty}{ }_{k \uparrow} M_{N_{k}}^{\tau}(\omega)=\mathbb{E}\left[\left|X_{\tau}\right|^{q}\right]\right\}\right]=1,
$$

where $N_{k}:=k \operatorname{lcm}(\mathcal{T})$ is a multiple of $\operatorname{lcm}(\mathcal{T})$. Consistency of estimation is the only property that supports the use of empirical moments and means, in a nutshell, that empirical moments converges to moments as the number of data points increases indefinitely. We stress that moments estimation requires that the range of existing moments is known. Otherwise, one runs the risk of evaluating scaling functions beyond the range of validity of moments scaling laws obtaining possible misleading results, such as spurious multifractality. In this respect, one must be aware that the empirical moment ${ }^{q} M_{N}^{\tau}(\omega)$ is well-defined for all $q \geq 0$ and does not allow to distinguish those $q$ for which $\mathbb{E}\left[\left|X_{\tau}\right|^{q}\right]<\infty$ from those for which $\mathbb{E}\left[\left|X_{\tau}\right|^{q}\right]=\infty$.

\section{The case of self-similar Lévy processes}

A Lévy process $\left\{X_{t}\right\}_{t \geq 0}$ is a stochastic process with stationary independent increments such that $X_{0}=0$. Independent increments means that the increments of the process over non-overlapping time windows are independent variables. It is well-known that a Lévy process $\left\{X_{t}\right\}_{t \geq 0}$ is self-similar if and only if the distribution of $X_{t}$ is strictly stable [33]. Strictly stability is expressed in terms of characteristic functions as

$$
\mathbb{E}\left[\exp \left(i k X_{t}\right)\right]=\exp [-t \psi(k)],
$$

$\S$ As $X_{\tau+1}=\sum_{n=0}^{\tau} \Delta_{n}^{1} X$ and $\left|\sum_{n=0}^{\tau} \delta_{n}\right|^{q} \leq 2^{q \tau} \cdot \sum_{n=0}^{\tau}\left|\delta_{n}\right|^{q}$ for all $q \geq 0$ and $\delta_{0}, \ldots, \delta_{\tau}$, the equality in law $\Delta_{n}^{1} X \stackrel{d}{=} X_{1}$ gives that $\mathbb{E}\left[\left|X_{\tau}\right|^{q}\right]<\infty$ for each integer $\tau$ if and only if $\mathbb{E}\left[\left|X_{1}\right|^{q}\right]<\infty$. 
where, with admissible parameters $0<\alpha \leq 2, \sigma>0$, and $-1 \leq \gamma \leq 1$ when $\alpha \neq 1$ and any $\gamma$ if $\alpha=1$, the function $\psi$ is defined as $\|$

$$
\psi(k):= \begin{cases}\sigma^{\alpha}|k|^{\alpha}\left[1-i \gamma \tan \left(\frac{\pi \alpha}{2}\right) \operatorname{sgn}(k)\right] & \text { if } \alpha \neq 1 ; \\ \sigma|k|-i \gamma k & \text { if } \alpha=1 .\end{cases}
$$

The parameter $\alpha$ is called the stability index and determines the scaling exponent $H$ associated to a self-similar Lévy process as $H=1 / \alpha$. When $\alpha=2$ the distribution of $X_{t}$ reduces to a Gaussian distribution and the process $\left\{X_{t}\right\}_{t \geq 0}$ becomes a version of the Brownian motion. Parameters $\sigma$ and $\gamma$ are a scale parameter and a skewness parameter, respectively. If $\gamma=0$, then $-X_{t}$ is distributed as $X_{t}$ for each $t \geq 0$ and the self-similar Lévy process is said to be symmetric.

\subsection{Main properties}

For the purposes of the present work, the most important feature of a self-similar Lévy process $\left\{X_{t}\right\}_{t \geq 0}$ with stability index $\alpha$ is that the distribution of $X_{t}$ displays fat tails with tail index $\alpha$ when $\alpha<2$ and $t>0$. Hereafter we implicitly assume that $\alpha<2$. A power-law decay with exponent $\alpha$ of both the right tail distribution $\mathbb{P}\left[X_{t}>x\right]$ and the left tail distribution $\mathbb{P}\left[X_{t}<-x\right]$ can be immediately found out in the simplest case $\alpha=1$, since in this case $\mathbb{1}$

$$
\mathbb{P}\left[X_{t}>x\right]=\mathbb{P}\left[X_{t}<-x\right]=\frac{1}{2}-\frac{1}{\pi} \arctan \left(\frac{x-t \gamma}{t \sigma}\right) \sim \frac{2 t}{\pi} \frac{\sigma}{x} .
$$

The case $\alpha \neq 1$ is a little bit more involved as the right tail distribution shows a fat tail with tail index $\alpha$ only if $-1<\gamma \leq 1$ (see [34, Theorem 2.4.2 if $\alpha<1$ and Corollary 2 of Theorem 2.5.1 if $\alpha>1$ ). On the contrary, if $\gamma=-1$, then $\mathbb{P}\left[X_{t}>x\right]=0$ for all $x>0$ when $\alpha<1$ or $\mathbb{P}\left[X_{t}>x\right]$ shrinks exponentially with $x>0$ when $\alpha>1$ (see [34], Theorem 2.5.2). For $\gamma>-1$ we exactly find

$$
\mathbb{P}\left[X_{t}>x\right] \sim \frac{t}{\pi}(1+\gamma) \Gamma(\alpha) \sin \left(\frac{\pi \alpha}{2}\right)\left(\frac{\sigma}{x}\right)^{\alpha},
$$

where $\Gamma$ is the Euler's gamma function. Symmetrically, the left tail distribution decays as a power law with exponent $\alpha$ if $-1 \leq \gamma<1$, whereas $\mathbb{P}\left[X_{t}<-x\right]=0$ for each $x>0$ or $\mathbb{P}\left[X_{t}<-x\right]$ decreases exponentially with $x>0$ according as $\alpha<1$ or $\alpha>1$ if $\gamma=1$. When $\gamma<1$ we have that

$$
\mathbb{P}\left[X_{t}<-x\right] \sim \frac{t}{\pi}(1-\gamma) \Gamma(\alpha) \sin \left(\frac{\pi \alpha}{2}\right)\left(\frac{\sigma}{x}\right)^{\alpha} .
$$

In spite of the complexity introduced by the skewness parameter $\gamma$, combining all together these results we recognise that the plain power law

$$
\mathbb{P}\left[\left|X_{t}\right|>x\right]=\mathbb{P}\left[X_{t}>x\right]+\mathbb{P}\left[X_{t}<-x\right] \sim \frac{t c}{x^{\alpha}}
$$

$\|$ As usual, $\operatorname{sgn}(k)$ denotes the $\operatorname{sign}$ of $k: \operatorname{sgn}(k):=1$ if $k \geq 0$ and $\operatorname{sgn}(k):=-1$ if $k<0$.

I If $f$ and $g$ are real functions of a real variable $x$ defined for all large enough $x, f(x) \sim g(x)$ means that $\lim _{x \uparrow \infty} f(x) / g(x)=1$. 
holds for all $\alpha<2$ and admissible value of other parameters with

$$
c:=\frac{2}{\pi} \Gamma(\alpha) \sin \left(\frac{\pi \alpha}{2}\right) \sigma^{\alpha}>0 .
$$

The consequence of (3i) is that $\mathbb{E}\left[\left|X_{t}\right|^{q}\right]<\infty$ for each $t>0$ if and only if $q<\alpha$.

Two other relevant features of the self-similar Lévy process $\left\{X_{t}\right\}_{t \geq 0}$ stem from the fact that the sequence $\left\{\Delta_{\tau n}^{\tau} X\right\}_{n \geq 0}$ of the increments over non-overlapping, consecutive time windows of size $\tau$ is a sequence of independent and identically distributed (i.i.d. for short) random variables. A first consequence of this fact is that the strong law of large numbers applies ensuring that for each integer temporal horizon $\mathcal{T}$ and non-negative order $q<\alpha$

$$
\mathbb{P}\left[\bigcap_{\tau=1}^{\mathcal{T}}\left\{\omega \in \Omega: \lim _{k \uparrow \infty}{ }^{q} M_{N_{k}}^{\tau}(\omega)=\mathbb{E}\left[\left|X_{\tau}\right|^{q}\right]=\mu(q) \cdot \tau^{\nu(q)}\right\}\right]=1,
$$

where $\mu(q)=\mathbb{E}\left[\left|X_{1}\right|^{q}\right]$ and $\nu(q)=q / \alpha$. This means that the estimation scheme previously discussed is consistent on the one hand, and that empirical moments inherit the monofractal nature of moments for orders smaller than the stability index on the other hand. Another consequence of the fact that $\left\{\Delta_{\tau n}^{\tau} X\right\}_{n \geq 0}$ is an i.i.d. sequence is a simple equality in law relating the estimators ${ }^{q} M_{N}^{\tau}$ and ${ }^{q} M_{N}^{1}$. Indeed, the self-similarity of the process $\left\{X_{t}\right\}_{t \geq 0}$ yields $\Delta_{\tau n}^{\tau} X \stackrel{d}{=} \Delta_{0}^{\tau} X \stackrel{d}{=} X_{\tau} \stackrel{d}{=} \tau^{1 / \alpha} X_{1} \stackrel{d}{=} \tau^{1 / \alpha} \Delta_{n}^{1} X$ for all $n \geq 0$. Then, bearing in mind the definition (2) of the estimator ${ }^{q} M_{N}^{\tau}$, we find for each non negative $q$, integer $\tau \leq \mathcal{T}$, and $N$ multiple of $\operatorname{lcm}(\mathcal{T})$ the equality in law

$$
{ }^{q} M_{N}^{\tau} \stackrel{d}{=} \frac{\tau^{q / \alpha}}{N / \tau} \sum_{n=0}^{N / \tau-1}\left|\Delta_{n}^{1} X\right|^{q}=\tau^{q / \alpha} \cdot{ }^{q} M_{N / \tau}^{1} .
$$

\subsection{Evidence of apparent multifractality}

Focusing on symmetric self-similar Lévy processes, in [17 the authors probed the empirical analysis to evaluate the scaling function by means of numerical simulations. Mimicking practical situations where no a priori information is available, they pretended not to know the stability index $\alpha$ and attempted to estimate moments on synthetic time series for trial orders $q$ up to and above the threshold $\alpha$. Such an attempt is always possible as the estimator ${ }^{q} M_{N}^{\tau}$ is well-defined for each non negative $q$ but estimation is phony for $q \geq \alpha$ as $\mathbb{E}\left[\left|X_{\tau}\right|^{q}\right]=\infty$ for all $\tau>0$ in this case. Surprisingly, the authors found that empirical moments of any order obey an approximate scaling law in time with a resulting scaling function which is concave and independent of the sample. They argued that such a scaling property becomes exact in the limit of long time series and extrapolated the piecewise-linear empirical scaling function

$$
\nu_{e}(q):= \begin{cases}q / \alpha & \text { if } q<\alpha \\ 1 & \text { if } q \geq \alpha .\end{cases}
$$

The same observation was previously reported in [8]. While scaling laws at orders $q<\alpha$ were expected in view of (5), the unforeseen behaviour of empirical moments in the 
regime $q \geq \alpha$ was somehow ascribed to finite sample effects. The important lesson to be learnt from this study was that if one trusts that empirical moments correctly estimate moments of given trial orders for time series generated by self-similar Lévy processes of unknown stability index, then one is led to think that data correspond to a non-trivial multifractal process even though they do not. Apparent multifractality can then emerge when fat-tailed distributions are associated to data.

Understanding apparent multifractality of self-similar Lévy processes from a mathematical standpoint amounts to explain whether or not and why empirical moments exhibit scaling laws in time at all orders for almost all the time series generated by the process. As a first speculative contribution to the problem, the distribution of the estimator ${ }^{q} M_{N}^{\tau}$ was investigated in [20] for $q>\alpha$. The idea was resorting to the norming constant $a_{N}:=N^{q / \alpha-1}$ in order to get in the large $N$ limit at a non-degenerate distribution for ${ }^{q} M_{N}^{\tau} / a_{N}$ whose dependence on $\tau$ may be disclosed. The following theorem reports and completes with the case $q=\alpha$ the convergence in distribution presented in [20]. The proof is provided in the next paragraph.

Theorem 1. Let $\left\{X_{t}\right\}_{t \geq 0}$ be a a self-similar Lévy process of stability index $\alpha<2$ defined on some probability space $(\Omega, \mathcal{F}, \mathbb{P})$ and let $c$ be the constant defined by 囵). Let $\tau \leq \mathcal{T}$ be positive integers and let $N_{k}:=k \operatorname{lcm}(\mathcal{T})$ be a multiple of $\operatorname{lcm}(\mathcal{T})$. Then

$$
{ }^{q} M_{N_{k}}^{\tau}-c \tau \ln \left(\frac{N_{k}}{\tau}\right) \stackrel{d}{\longrightarrow} \tau \cdot Z_{q}
$$

as $k \uparrow \infty$ if $q=\alpha$ and

$$
N_{k}^{1-q / \alpha} \cdot{ }^{q} M_{N_{k}}^{\tau} \stackrel{d}{\longrightarrow} \tau \cdot Z_{q}
$$

as $k \uparrow \infty$ if $q>\alpha, Z_{q}$ being a non-degenerate stable variable of stability index $\alpha / q$ with law independent of $\tau$.

Theorem 1 shows that the distribution of ${ }^{q} M_{N}^{\tau} / a_{N}$ is endowed with well-defined scaling laws $\uplus$ in the size $\tau$ of time windows for orders $q$ strictly larger than $\alpha$, where no centering procedure is required to reach a non degenerate limit when $N$ is sent to infinite. Interestingly, the exponent involved in these laws coincides with that found in [8] and [17. However, despite the nice result, this theorem does not give reasoning for the behaviour of empirical moments observed in [17] since it only deals with their statistics over the different samples on the one hand and since it is not obvious that their own scaling in time may be deduced from scaling laws of their distribution on the other hand. Actually, we believe that the basic idea of employing a norming constant is not sufficient to explain apparent multifractality of self-similar Lévy processes. The reason is that, contrary to the case of distributions, when $q \geq \alpha$ no norming constant $a_{N}$ exists with the property that for almost all $\omega \in \Omega$ the normed empirical moment

+ Similar results were also obtained for the fractional Lévy motion [20] and for processes with more general fat-tailed independent [21] and weakly dependent [25] increments within a special estimation scheme where, for a given $\xi \in(0,1), \tau$ grows as $N^{\xi}$ as $N$ increases. In [21] and [25] convergence in distribution was improved to convergence in probability. 
${ }^{q} M_{N}^{\tau}(\omega) / a_{N}$ attains at large $N$ a somehow useful limit to detect scaling laws. This fact is made evident by the following proposition, which is proven in the next paragraph.

Proposition 1. Assume that $\left\{X_{t}\right\}_{t \geq 0}$ is a self-similar Lévy process of stability index $\alpha<2$ defined on some probability space $(\Omega, \mathcal{F}, \mathbb{P})$. Let $\mathcal{T}$ be a positive integer and let $N_{k}:=k \operatorname{lcm}(\mathcal{T})$ be a multiple of $\operatorname{lcm}(\mathcal{T})$. Fix any $q \geq \alpha$ and let $\left\{a_{N_{k}}\right\}_{k \geq 1}$ be an increasing sequence of positive numbers. Then

$$
\mathbb{P}\left[\bigcap_{\tau=1}^{\mathcal{T}}\left\{\omega \in \Omega: \lim _{k \uparrow \infty} \frac{{ }^{q} M_{N_{k}}^{\tau}(\omega)}{a_{N_{k}}}=0\right\}\right]=1
$$

or

$$
\mathbb{P}\left[\bigcap_{\tau=1}^{\mathcal{T}}\left\{\omega \in \Omega: \limsup _{k \uparrow \infty} \frac{{ }^{q} M_{N_{k}}^{\tau}(\omega)}{a_{N_{k}}}=\infty\right\}\right]=1
$$

according as the series $\sum_{k=1}^{\infty}\left(k a_{N_{k}}\right)^{-\alpha / q}$ converges or diverges.

Proposition 1 states that, whatever $a_{N}$ is, if $q \geq \alpha$ either ${ }^{q} M_{N}^{\tau} / a_{N}$ converges a.s. to zero as $N$ is sent to infinite or it does not converge at all. As a consequence, no property of single empirical moments can be highlighted by resorting to a norming constant $a_{N}$ when $q \geq \alpha$, not even when $a_{N}$ coincides with $N^{q / \alpha-1}$ as in Theorem 1 .

\subsection{Proof of Theorem 1 and Proposition 1}

In order to prove Theorem 1 it is enough to show that there exists a non-degenerate stable variable $Z_{q}$ of stability index $\alpha / q$ such that

$$
{ }^{q} M_{N}^{1}-c \ln (N) \stackrel{d}{\longrightarrow} Z_{q}
$$

as $N \uparrow \infty$ if $q=\alpha$ and

$$
N^{1-q / \alpha} \cdot{ }^{q} M_{N}^{1} \stackrel{d}{\longrightarrow} Z_{q}
$$

as $N \uparrow \infty$ if $q>\alpha$. Indeed, since $\left\{N_{k} / \tau\right\}_{k \geq 1}$ is a subsequence of the integers for a given $\tau \leq \mathcal{T}$, combining (6) with (8) and (9) we get that

$$
{ }^{q} M_{N_{k}}^{\tau}-c \tau \ln \left(\frac{N_{k}}{\tau}\right) \stackrel{d}{=} \tau \cdot\left[{ }^{q} M_{N_{k} / \tau}^{1}-c \ln \left(N_{k} / \tau\right)\right] \stackrel{d}{\longrightarrow} \tau \cdot Z_{q}
$$

as $k \uparrow \infty$ if $q=\alpha$ and

$$
N_{k}^{1-q / \alpha} \cdot{ }^{q} M_{N_{k}}^{\tau} \stackrel{d}{=} \tau \cdot\left(N_{k} / \tau\right)^{1-q / \alpha} \cdot{ }^{q} M_{N_{k} / \tau}^{1} \stackrel{d}{\longrightarrow} \tau \cdot Z_{q}
$$

as $k \uparrow \infty$ if $q>\alpha$. The law of $Z_{q}$ is clearly independent of $\tau$ as ${ }^{q} M_{N}^{1}$ does not depend on it.

Convergences in distribution (8) and (9) follow from standard limit theory for sums of i.i.d. random variables. We point out that $\left|X_{1}\right|^{q}$ belongs to the normal domain of attraction of a stable variable with stability index $\alpha / q \leq 1$ when $q \geq \alpha$ (see [35], concluding remark in Chapter XVII.5). Indeed, $\left|X_{1}\right|^{q}$ is positive and $\lim _{x \uparrow \infty} x^{\alpha / q} \cdot \mathbb{P}\left[\left|X_{1}\right|^{q}>x\right]=c$ from (3) . Then, since the increments $\left\{\Delta_{n}^{1} X\right\}_{n \geq 0}$ over consecutive, non-overlapping time windows of size 1 form a sequence of independent 
variables distributed as $X_{1}$ for Lévy processes, we realise that (see [35], Theorem 3 in Chapter XVII.5)

$$
{ }^{q} M_{N}^{1}-b_{N}=\frac{1}{N} \sum_{n=0}^{N-1}\left|\Delta_{n}^{1} X\right|^{q}-b_{N} \stackrel{d}{\longrightarrow} Z_{q}
$$

as $N \uparrow \infty$ if $q=\alpha$ and

$$
N^{1-q / \alpha} \cdot{ }^{q} M_{N}^{1}=\frac{1}{N^{\frac{q}{\alpha}}} \sum_{n=0}^{N-1}\left|\Delta_{n}^{1} X\right|^{q} \stackrel{d}{\longrightarrow} Z_{q}
$$

as $N \uparrow \infty$ if $q>\alpha$, where $Z_{q}$ is a non-degenerate stable variable of stability index $\alpha / q$ and $\left\{b_{N}\right\}_{N \geq 1}$ is any numerical sequence such that

$$
\lim _{N \uparrow \infty}\left\{\mathbb{E}\left[N \sin \left(\left|X_{1}\right|^{\alpha} / N\right)\right]-b_{N}\right\}
$$

exists finite. A centering procedure is required when $q=\alpha$ to avoid a degenerate limit distribution. The following lemma, whose proof is reported in Appendix A, states that $b_{N}$ can be taken equal to $c \ln (N)$ for each $N \geq 1, c$ being the constant defined by (4). Theorem 1 is thus demonstrated.

Lemma 1. Let $c$ be the constant defined by (4). Then

$$
\lim _{N \uparrow \infty}\left\{\mathbb{E}\left[N \sin \left(\left|X_{1}\right|^{\alpha} / N\right)\right]-c \ln (N)\right\}
$$

exists finite.

Proposition 1 is an immediate consequence of the following important result due to Feller (see [36], Theorem 2.5.9).

Theorem 2. Let $\left\{V_{n}\right\}_{n \geq 0}$ be a sequence of i.i.d. random variables defined on some probability space $(\Omega, \mathcal{F}, \mathbb{P})$ and let $\left\{b_{N}\right\}_{N \geq 1}$ be an increasing sequence of positive numbers. If $\mathbb{E}\left[\left|V_{0}\right|\right]=\infty$, then

$$
\lim _{N \uparrow \infty} \frac{1}{N b_{N}} \sum_{n=0}^{N-1} V_{n}=0 \quad \text { a.s. }
$$

or

$$
\limsup _{N \uparrow \infty} \frac{1}{N b_{N}}\left|\sum_{n=0}^{N-1} V_{n}\right|=\infty \quad \text { a.s. }
$$

according as the series $\sum_{N=1}^{\infty} \mathbb{P}\left[\left|V_{0}\right|>N b_{N}\right]$ converges or diverges.

In order to prove Proposition 1 we fix $\tau \leq \mathcal{T}$, we set $\lambda:=\operatorname{lcm}(\mathcal{T}) / \tau$, and we group the first $N_{k} / \tau=\lambda k$ increments of the process over time windows of size $\tau$ into $k$ consecutive blocks of $\lambda$ elements each. This allows us to recast ${ }^{q} M_{N_{k}}^{\tau}$ as

$$
{ }^{q} M_{N_{k}}^{\tau}=\frac{1}{\lambda k} \sum_{n=0}^{\lambda k-1}\left|\Delta_{\tau n}^{\tau} X\right|^{q}=\frac{1}{k} \sum_{i=0}^{k-1} V_{i}
$$


with

$$
V_{i}:=\frac{1}{\lambda} \sum_{j=0}^{\lambda-1}\left|\Delta_{\tau(\lambda i+j)}^{\tau} X\right|^{q} .
$$

Since $\left\{\Delta_{\tau n}^{\tau} X\right\}_{n \geq 0}$ is a sequence of i.i.d. random variables, $\left\{V_{i}\right\}_{i \geq 0}$ is a sequence of i.i.d. positive random variables. Moreover, as the $\left|\Delta_{\tau n}^{\tau} X\right|^{q}$ 's are fat-tailed variables distributed as $\left|X_{\tau}\right|^{q}$, for the sum of the first $\lambda$ of them we have that (see [35], last corollary in Chapter VIII.8)

$$
\mathbb{P}\left[\sum_{j=0}^{\lambda-1}\left|\Delta_{\tau j}^{\tau} X\right|^{q}>x\right] \sim \lambda \mathbb{P}\left[\left|X_{\tau}\right|^{q}>x\right] .
$$

Thus, bearing in mind (3) , we find that

$$
\mathbb{P}\left[V_{0}>x\right]=\mathbb{P}\left[\sum_{j=0}^{\lambda-1}\left|\Delta_{\tau j}^{\tau} X\right|^{q}>\lambda x\right] \sim \lambda \mathbb{P}\left[\left|X_{\tau}\right|^{q}>\lambda x\right] \sim \frac{\tau c \lambda^{1-\alpha / q}}{x^{\alpha / q}}
$$

with the consequence that for each increasing sequence $\left\{a_{N_{k}}\right\}_{k \geq 1}$ of positive numbers the series $\sum_{k=1}^{\infty} \mathbb{P}\left[V_{0}>k a_{N_{k}}\right]$ converges or diverges if and only if the series $\sum_{k=1}^{\infty}\left(k a_{N_{k}}\right)^{-\alpha / q}$ does. When $q \geq \alpha$, then $\mathbb{E}\left[V_{0}\right]=\infty$ and Theorem 2 applies stating that for a given increasing sequence $\left\{a_{N_{k}}\right\}_{k \geq 1}$ of positive numbers

$$
\lim _{k \uparrow \infty} \frac{{ }^{q} M_{N_{k}}^{\tau}}{a_{N_{k}}}=\lim _{k \uparrow \infty} \frac{1}{k a_{N_{k}}} \sum_{i=0}^{k-1} V_{i}=0 \quad \text { a.s. }
$$

or

$$
\limsup _{k \uparrow \infty} \frac{{ }^{q} M_{N_{k}}^{\tau}}{a_{N_{k}}}=\infty \quad \text { a.s. }
$$

according as the series $\sum_{k=1}^{\infty} \mathbb{P}\left[V_{0}>k a_{N_{k}}\right]$, and hence $\sum_{k=1}^{\infty}\left(k a_{N_{k}}\right)^{-\alpha / q}$, converges or diverges. As $\tau$ is arbitrary and as the intersection of a finite number of events with probability measure one has probability measure one too, Proposition 1 is proven.

\subsection{Almost sure apparent multifractality}

Numerical investigations carried out in [17] lead to the conjecture that, irrespective of the order $q$, for almost all $\omega \in \Omega$ the empirical moment ${ }^{q} M_{N}^{\tau}(\omega)$ becomes proportional to $\tau^{\nu_{e}(q)}$ at large $N$ with coefficient of proportionality independent of $\tau, \nu_{e}(q)$ being the empirical scaling function (77). Within this scenario the coefficient of proportionality should be equal to ${ }^{q} M_{N}^{1}(\omega)$. Then, we believe that a breakthrough in understanding apparent multifractality of self-similar Lévy processes may come from the study of the ratio ${ }^{q} M_{N}^{\tau} /{ }^{q} M_{N}^{1}$, with the purpose of demonstrating that it converges a.s. to $\tau^{\nu_{e}(q)}$ as $N$ is sent to infinite. Actually, this is an evident fact at orders $q$ smaller than the stability index $\alpha$, where the strong law of large numbers works. In contrast, it requires to hold in the regime $q \geq \alpha$ that a nontrivial decoupling between the randomness source $\omega$ and the size $\tau$ of time windows occurs at large $N$ as for almost all $\omega$ the empirical moment ${ }^{q} M_{N}^{\tau}(\omega)$ remains a fluctuating quantity depending on the specific sample $\omega$, 
whose behaviour cannot be regularised by the introduction of a norming constant as stated by Proposition 1 .

In this paper we show that the conjecture holds for self-similar Lévy processes. The reason of the decoupling between the randomness source $\omega$ and the variable $\tau$ at orders $q \geq \alpha$ lies, in a nutshell, in the fact that large values of the increments of the process over time windows of integer size $\tau$ are due to large fluctuations of only one of the $\tau$ corresponding unitary increments. We let the following theorem to state our main result.

Theorem 3. Let $\left\{X_{t}\right\}_{t \geq 0}$ be a self-similar Lévy process of stability index $\alpha<2$ defined on some probability space $(\Omega, \mathcal{F}, \mathbb{P})$ and let $\nu_{e}$ be the empirical scaling function (7). Let $\mathcal{T}$ be a positive integer and let $N_{k}:=k \operatorname{lcm}(\mathcal{T})$ be a multiple of $\operatorname{lcm}(\mathcal{T})$. Then

$$
\mathbb{P}\left[\bigcap_{\tau=1}^{\mathcal{T}}\left\{\omega \in \Omega: \lim _{k \uparrow \infty} \frac{{ }^{q} M_{N_{k}}^{\tau}(\omega)}{{ }^{q} M_{N_{k}}^{1}(\omega)}=\tau^{\nu e}(q)\right\}\right]=1
$$

for all $q \geq 0$.

The theorem demonstrates that empirical moments possess their own scaling law in time at any order $q$ for almost all the sample paths of self-similar Lévy processes. This fact leads to the systematic emergence of apparent multifractality and completely explains the numerical findings of [8] and [17]. Apparent multifractality emerges a.s. even when $q=\alpha$, although Theorem 1 tells us that the distribution of estimators does not scale with time in this case. We stress that the scaling law of empirical moments needed to be disclosed of an appropriate stochastic normalisation, which was able to balance fluctuations without hiding the dependence on time. Theorem 3 states that such a stochastic normalisation is exactly ${ }^{q} M_{N}^{1}$.

Theorem 3 is an immediate consequence of (5) when $q<\alpha$. In order to prove the theorem in the case $q \geq \alpha$ we observe that, given $N$ multiple of $\operatorname{lcm}(\mathcal{T})$ and grouping the first $N$ unitary increments of the process into $N / \tau$ consecutive blocks of $\tau \leq \mathcal{T}$ elements each, the estimator ${ }^{q} M_{N}^{1}$ can be rewritten as

$$
{ }^{q} M_{N}^{1}=\frac{1}{N} \sum_{n=0}^{N-1}\left|\Delta_{n}^{1} X\right|^{q}=\frac{1}{N} \sum_{n=0}^{N / \tau-1} \sum_{i=0}^{\tau-1}\left|\Delta_{\tau n+i}^{1} X\right|^{q},
$$

so that

$$
\frac{{ }^{q} M_{N}^{\tau}}{{ }^{q} M_{N}^{1}}=\tau \cdot \frac{\sum_{n=0}^{N / \tau-1}\left|\Delta_{\tau n}^{\tau} X\right|^{q}}{\sum_{n=0}^{N / \tau-1} \sum_{i=0}^{\tau-1}\left|\Delta_{\tau n+i}^{1} X\right|^{q}} .
$$

Then, since $\left\{N_{k} / \tau\right\}_{k \geq 1}$ is a subsequence of the integers for $\tau \leq \mathcal{T}$ and since a finite intersection of measurable sets with probability measure one has probability measure one too, Theorem 3 descends from the following proposition as $\nu_{e}(q)=1$ for each $q \geq \alpha$.

Proposition 2. Assume that $\left\{X_{t}\right\}_{t \geq 0}$ is a self-similar Lévy process of stability index $\alpha<2$ defined on some probability space $(\Omega, \mathcal{F}, \mathbb{P})$. Fix any $q \geq \alpha$ and integer $\tau \geq 1$. Then

$$
\lim _{N \uparrow \infty} \frac{\sum_{n=0}^{N-1}\left|\Delta_{\tau n}^{\tau} X\right|^{q}}{\sum_{n=0}^{N-1} \sum_{i=0}^{\tau-1}\left|\Delta_{\tau n+i}^{1} X\right|^{q}}=1 \quad \text { a.s. }
$$


Proposition 2 is interesting by its own and could be easily extended to processes with more general stationary independent fat-tailed increments. Its proof is outlined in the next paragraph. This proposition states that the large fluctuations of $\left|\Delta_{\tau n}^{\tau} X\right|^{q}$ are comparable to those of the sum $\sum_{i=0}^{\tau-1}\left|\Delta_{\tau n+i}^{1} X\right|^{q}$ referring to the same time windows, due to the hypothesis of independent increments. We immediately recognise that this fact is possible only if a unique unitary increment dominates by noticing that $\Delta_{\tau n}^{\tau} X=\sum_{i=0}^{\tau-1} \Delta_{\tau n+i}^{1} X$. Thus, it is confirmed that large values of the increments of the process over time windows of size $\tau$ are ascribed to large fluctuations of only one of the corresponding unitary increments if $q \geq \alpha$.

\subsection{Proof of Proposition 2}

We outline the proof of Proposition 2 postponing technical details in the appendices with the purpose of not interrupting the flow of the presentation. We fix once and for all a moment order $q \geq \alpha$ and, noticing that the instance $\tau=1$ is trivial, an integer $\tau \geq 2$. Since we found convenient reducing the proof to a comparison among extreme events, at first we consider for each $n \geq 0$ the pointwise largest and second largest of the $\tau$ unitary increments $\left|\Delta_{\tau n}^{1} X\right|^{q},\left|\Delta_{\tau n+1}^{1} X\right|^{q}, \ldots,\left|\Delta_{\tau n+\tau-1}^{1} X\right|^{q}$, denoted by $U_{n}$ and $V_{n}$ respectively. Both $\left\{U_{n}\right\}_{n \geq 0}$ and $\left\{V_{n}\right\}_{n \geq 0}$ are sequences of i.i.d. positive random variables due the hypothesis of stationary independent increments. We observe that $U_{0}$ is not larger than $x$ if and only if all the variables $\left|\Delta_{0}^{1} X\right|^{q},\left|\Delta_{1}^{1} X\right|^{q}, \ldots,\left|\Delta_{\tau-1}^{1} X\right|^{q}$ are smaller than or equal to $x$, so that $\mathbb{P}\left[U_{0} \leq x\right]=\mathbb{P}^{\tau}\left[\left|\Delta_{0}^{1} X\right|^{q} \leq x\right]=\mathbb{P}^{\tau}\left[\left|X_{1}\right|^{q} \leq x\right]$ and $\mathbb{P}\left[U_{0}>x\right] \sim \tau \mathbb{P}\left[\left|X_{1}\right|^{q}>x\right]$ follows. Similarly, $V_{0}$ is not larger than $x$ if and only if at least $\tau-1$ among these variables are smaller than or equal to $x$, with the consequence that $\mathbb{P}\left[V_{0} \leq x\right]=\mathbb{P}^{\tau}\left[\left|X_{1}\right|^{q} \leq x\right]+\tau \mathbb{P}\left[\left|X_{1}\right|^{q}>x\right] \mathbb{P}^{\tau-1}\left[\left|X_{1}\right|^{q} \leq x\right]$, providing $\mathbb{P}\left[V_{0}>x\right] \sim(1 / 2) \tau(\tau-1) \mathbb{P}^{2}\left[\left|X_{1}\right|^{q}>x\right]$. The asymptotic formulas reflect a simple combinatorial argument: large fluctuations of $U_{0}$ require large values of only one increment among $\left|\Delta_{0}^{1} X\right|^{q},\left|\Delta_{1}^{1} X\right|^{q}, \ldots,\left|\Delta_{\tau-1}^{1} X\right|^{q}$, while large fluctuations of $V_{0}$ demand large values of at least two of them. Combining these formulas with (3) we obtain that

$$
\mathbb{P}\left[U_{0}>x\right] \sim \frac{\tau c}{x^{\beta}}
$$

and

$$
\mathbb{P}\left[V_{0}>x\right] \sim \frac{\tau(\tau-1)}{2} \frac{c^{2}}{x^{2 \beta}}
$$

where $\beta:=\alpha / q \leq 1$ and $c$ is given by (41). The following lemma, which is proven in Appendix B, brings the $U_{n}$ 's and $V_{n}$ 's up showing that in order to prove Proposition 2 it is enough to demonstrate that the ratio

$$
R_{N}:=\frac{\sum_{n=0}^{N-1} V_{n}}{\sum_{n=0}^{N-1} U_{n}}
$$

goes to zero a.s. as $N$ is sent to infinite. We notice that $\sum_{n=0}^{N-1} U_{n}=0$ if and only if $\Delta_{n}^{1} X=0$ for each $n \leq \tau N-1$ and that both these events have probability measure equal to zero since the distribution of $X_{1}$ does not put any finite mass on any point. 
Lemma 2. There exists a positive constant $h$, in general depending on both $q$ and $\tau$, such that

$$
\left|\frac{\sum_{n=0}^{N-1}\left|\Delta_{\tau n}^{\tau} X\right|^{q}}{\sum_{n=0}^{N-1} \sum_{i=0}^{\tau-1}\left|\Delta_{\tau n+i}^{1} X\right|^{q}}-1\right| \leq h \cdot\left(R_{N}\right)^{e} \quad \text { a.s. }
$$

for all $N \geq 1$ with $e:=\min \{1,1 / q\}$.

The fact that $\lim _{N \uparrow \infty} R_{N}=0$ a.s. is not surprising from the point of view of intuition. As large fluctuations of the $V_{n}$ 's are much less probable than comparable fluctuations of the $U_{n}$ 's, one expects that the numerator of $R_{N}$ becomes smaller and smaller in comparison with the denominator if $N$ becomes larger and larger. However, one has to pay attention that there are subtle samples for which the denominator of $R_{N}$ does not grow quickly enough to dominate the numerator, with the consequence that $R_{N}$ is not negligible in the large $N$ limit. These samples have thus to be isolated. Fortunately, they constitute a set which has a smaller and smaller probability measure as $N$ is let to increase. These considerations prompt us to resort to the following strategy to prove that $\lim _{N \uparrow \infty} R_{N}=0$ a.s. . For each $N \geq 1$ we pick a positive number $\lambda_{N}$, which is for the moment unknown. Then we consider the set $E$ of all $\omega \in \Omega$ with the property that there exists $N_{0} \geq 1$ such that $\sum_{n=0}^{N-1} U_{n}(\omega)>\lambda_{N}$ if $N \geq N_{0}$. This set will correspond to the set of samples for which the denominator of $R_{N}$ is able to dominate the numerator. Finally we search for the $\lambda_{N}$ 's that makes $E$ a set with probability measure equal to one on the one hand, and allow to satisfy $\lim _{N \uparrow \infty} R_{N}(\omega)=0$ for almost all $\omega \in E$ on the other hand. In order to put into practice this strategy, for each $N \geq 1$ we introduce the measurable set

$$
E_{N}:=\left\{\omega \in \Omega: \sum_{n=0}^{N-1} U_{n}(\omega)>\lambda_{N}\right\} .
$$

The set $E$ of all $\omega \in \Omega$ for which there exists $N_{0} \geq 1$ with the property that $\sum_{n=0}^{N-1} U_{n}(\omega)>\lambda_{N}$ if $N \geq N_{0}$ is nothing but the limit inferior of the sequence of the $E_{N}$ 's: $E=\bigcup_{n=1}^{\infty} \bigcap_{N=n}^{\infty} E_{N}$. Indeed, if $\omega \in E$ one can find $N_{0} \geq 1$ so that $\omega \in \bigcap_{N=N_{0}}^{\infty} E_{N}$ and vice versa. We also introduce the measurable set

$$
F:=\left\{\omega \in \Omega: \lim _{N \uparrow \infty} \frac{1}{\lambda_{N}} \sum_{n=0}^{N-1} V_{n}(\omega)=0\right\}
$$

and observe that $\lim _{N \uparrow \infty} R_{N}(\omega)=0$ if $\omega \in E \cap F$. Indeed, both the conditions $0 \leq R_{N}(\omega)<\left(1 / \lambda_{N}\right) \sum_{n=0}^{N-1} V_{n}(\omega)$ for all $N \geq N_{0}$ with some $N_{0} \geq 1$ and $\lim _{N \uparrow \infty}\left(1 / \lambda_{N}\right) \sum_{n=0}^{N-1} V_{n}(\omega)=0$ are satisfied when $\omega \in E \cap F$. Thus, if a choice of the $\lambda_{N}$ 's for which both $\mathbb{P}[E]=1$ and $\mathbb{P}[F]=1$ exists, then $\lim _{N \uparrow \infty} R_{N}=0$ a.s. follows.

A straight application of the Borel-Cantelli Lemma yields that $\mathbb{P}\left[E^{c}\right]=0$, and hence $\mathbb{P}[E]=1$, if the condition

$$
\sum_{N=1}^{\infty} \mathbb{P}\left[E_{N}^{c}\right]=\sum_{N=1}^{\infty} \mathbb{P}\left[\sum_{n=0}^{N-1} U_{n} \leq \lambda_{N}\right]<\infty
$$

is met. This amounts to say that the event where the denominator of $R_{N}$ is not able to dominate the numerator is unlikely enough at large $N$. We let the following lemma, 
which is demonstrated in Appendix C, to introduce a choice of the $\lambda_{N}$ 's that fulfils such condition.

Lemma 3. There exist two strictly positive constants $k$ and $K$, in general depending on both $q$ and $\tau$, such that

$$
\mathbb{P}\left[\sum_{n=0}^{N-1} U_{n} \leq \lambda_{N}\right] \leq \frac{K}{N^{2}}
$$

for all $N \geq 1$ with $\beta:=\alpha / q$ and

$$
\lambda_{N}:= \begin{cases}k N^{\frac{1}{\beta}}(\ln N+1)^{-\frac{1-\beta}{\beta}} & \text { if } \beta<1 \\ k N(\ln N+1) & \text { if } \beta=1 .\end{cases}
$$

The $\lambda_{N}$ 's introduced by the lemma not only satisfy the condition (14), in such a way that $\mathbb{P}[E]=1$, but even give that $\mathbb{P}[F]=1$. Indeed, if the positive variable $V_{0}$ possesses finite expected value, namely if $q<2 \alpha$ as stated by (13), then the strong law of the large numbers applies ensuring that a measurable set $G$ exists with the properties that $\mathbb{P}[G]=1$ and $\lim _{N \uparrow \infty}(1 / N) \sum_{n=0}^{N-1} V_{n}(\omega)=\mathbb{E}\left[V_{0}\right]$ for all $\omega \in G$. It follows that $\lim _{N \uparrow \infty}\left(1 / \lambda_{N}\right) \sum_{n=0}^{N-1} V_{n}(\omega)=0$ for each $\omega \in G$ since $N / \lambda_{N}$ approaches zero as $N$ is sent to infinite. Thus $G \subseteq F$ and $\mathbb{P}[F]=1$. If instead $V_{0}$ does not possess finite expected value, namely if $q \geq 2 \alpha$, then Theorem 2 applies yielding that $\lim _{N \uparrow \infty}\left(1 / \lambda_{N}\right) \sum_{n=0}^{N-1} V_{n}=0$ a.s. since one can easily verify that $\left\{\lambda_{N} / N\right\}_{N \geq 1}$ is an increasing sequence of positive numbers and that $\sum_{N=1}^{\infty} \lambda_{N}^{-2 \beta}<\infty$. The latter fact amounts to $\sum_{N=1}^{\infty} \mathbb{P}\left[V_{0}>\lambda_{N}\right]<\infty$ thanks to (13). The proof of Proposition 2 is thus concluded.

\section{Conclusions}

In this paper we have proven that empirical moments of self-similar Lévy processes possess almost surely their own scaling laws with a piecewise-linear scaling function. Such scaling laws differ from those of the underlying process, as they extend the range of validity of the latter that is limited because of the presence of fat tails. Since only the scaling properties of empirical moments can be detected from a particular sample, our results explain the emergence of apparent multifractality in self-similar Lévy processes once and for all.

The main lesson to be learnt from this study is that inferring multifractality from scaling laws of empirical moments may lead to mistaken conclusions in many practical situations where fat-tailed variables are involved. Nonlinearities of the scaling function should not be taken as an evidence for genuine multifractality unless the range of existing moments is known, otherwise better tests for multiplicative noise should be developed to assess whether the data really account for such a property or not.

Results proven in this paper are concerned with processes with independent increments. As this condition is usually not met in real time series, dependence should be considered in future research in order to clarify the interplay between multifractality and 
heavy-tails for more general stochastic processes. The quest is particularly important for financial data, which often display fat tails and strong dependence, and which

produce nonlinear scaling functions at the same time. We expect that the use of a stochastic normalisation to regularise empirical moments, represented by the estimator of moments at a given size of time windows, can help to explain the emergence of apparent multifractality, if any, in a wide context. The particular case of self-similar Lévy processes constitutes an example in which such procedure succeeds.

\section{Acknowledgement}

The author is grateful to Fulvio Baldovin and Attilio Stella for stimulating discussions which led to the initiation of this study.

\section{Appendices}

\section{Appendix A. Proof of Lemma 1}

Applying the Fubini-Tonelli Theorem to the integral

$$
\int_{0}^{\infty} d x \int_{\Omega} \mathbb{P}[d \omega] \cos (x / N) \mathbb{1}\left(\left|X_{1}(\omega)\right|^{\alpha}>x\right)
$$

we get that

$$
\mathbb{E}\left[N \sin \left(\left|X_{1}\right|^{\alpha} / N\right)\right]=\int_{0}^{\infty} d x \cos (x / N) \mathbb{P}\left[\left|X_{1}\right|^{\alpha}>x\right] .
$$

Then, subtracting and adding the convergent Dirichlet-type integral $\int_{1}^{\infty} d x \frac{\cos (x / N)}{x}$ we can write

$$
\begin{aligned}
\mathbb{E}\left[N \sin \left(\left|X_{1}\right|^{\alpha} / N\right)\right]-c \ln (N) & =\int_{0}^{1} d x \cos (x / N) \mathbb{P}\left[\left|X_{1}\right|^{\alpha}>x\right] \\
& +\int_{1}^{\infty} d x \cos (x / N) \mathbb{P}\left[\left|X_{1}\right|^{\alpha}>x\right]-c \ln (N) \\
& =\int_{0}^{1} d x \cos (x / N) \mathbb{P}\left[\left|X_{1}\right|^{\alpha}>x\right] \\
& +\int_{1}^{\infty} d x \cos (x / N)\left\{\mathbb{P}\left[\left|X_{1}\right|^{\alpha}>x\right]-\frac{c}{x}\right\} \\
& +c\left\{\int_{1}^{\infty} d x \frac{\cos (x / N)}{x}-\ln (N)\right\} .
\end{aligned}
$$

Since we can find $d>0$ in such a way that $\left|\mathbb{P}\left[\left|X_{1}\right|>x\right]-c / x^{\alpha}\right| \leq d / x^{2 \alpha}$ for all $x \geq 1$ when $c$ is as in (44) (see [34], Theorem 2.4.2 if $\alpha<1$ and Corollary 2 of Theorem 2.5.1 if $\alpha>1$ ), the Dominated Convergence Theorem yields that the first and the second terms of (A.1) form convergent sequences. Thus, the trivial identity

$$
\int_{1}^{\infty} d x \frac{\cos (x / N)}{x}-\ln (N)=\int_{1 / N}^{1} d x \frac{\cos (x)-1}{x}+\int_{1}^{\infty} d x \frac{\cos (x)}{x}
$$


allows us to conclude that

$$
\begin{aligned}
\lim _{N \uparrow \infty}\left\{\mathbb{E}\left[N \sin \left(\left|X_{1}\right|^{\alpha} / N\right)\right]-c \ln (N)\right\} & =\int_{0}^{1} d x \mathbb{P}\left[\left|X_{1}\right|^{\alpha}>x\right] \\
& +\int_{1}^{\infty} d x\left\{\mathbb{P}\left[\left|X_{1}\right|^{\alpha}>x\right]-\frac{c}{x}\right\} \\
& +c\left\{\int_{0}^{1} d x \frac{\cos (x)-1}{x}+\int_{1}^{\infty} d x \frac{\cos (x)}{x}\right\} .
\end{aligned}
$$

\section{Appendix B. Proof of Lemma 2}

The proof of Lemma 2 relies on the following bounds, which hold for all numbers $0 \leq v \leq u$ and $\tau \geq 1$ with $h:=2 \tau$ or $h:=(q+2) \tau^{q}$ according as $q \leq 1$ or $q>1$ :

$$
\left[\max \left\{0, u^{\frac{1}{q}}-(\tau-1) v^{\frac{1}{q}}\right\}\right]^{q} \geq \begin{cases}u+(\tau-1) v-h v & \text { if } q \leq 1 \\ u+(\tau-1) v-h u^{1-\frac{1}{q}} v^{\frac{1}{q}} & \text { if } q>1\end{cases}
$$

and

$$
\left[u^{\frac{1}{q}}+(\tau-1) v^{\frac{1}{q}}\right]^{q} \leq \begin{cases}u+h v & \text { if } q \leq 1 \\ u+h u^{1-\frac{1}{q}} v^{\frac{1}{q}} & \text { if } q>1\end{cases}
$$

We shall prove these inequalities later. At the moment, we exploit them to show that for the given order $q>0$ and integer $\tau \geq 2$ and for each $N \geq 1$ and real numbers $\delta_{0}, \delta_{1}, \ldots, \delta_{\tau N-1}$ not all equal to zero

$$
1-h \cdot\left(\frac{\sum_{n=0}^{N-1} v_{n}}{\sum_{n=0}^{N-1} u_{n}}\right)^{e} \leq \frac{\sum_{n=0}^{N-1}\left|\sum_{i=0}^{\tau-1} \delta_{\tau n+i}\right|^{q}}{\sum_{n=0}^{N-1} \sum_{i=0}^{\tau-1}\left|\delta_{\tau n+i}\right|^{q}} \leq 1+h \cdot\left(\frac{\sum_{n=0}^{N-1} v_{n}}{\sum_{n=0}^{N-1} u_{n}}\right)^{e},
$$

where $u_{n}$ and $v_{n}$ are used to denote the largest and the second largest of the $\tau$ values $\left|\delta_{\tau n}\right|^{q},\left|\delta_{\tau n+1}\right|^{q}, \ldots,\left|\delta_{\tau n+\tau-1}\right|^{q}$ respectively and $e:=\min \{1,1 / q\}$. The lemma follows from this formula setting $\delta_{n}:=\Delta_{n}^{1} X(\omega)$, with $\omega \in \Omega$ chosen so that the $\Delta_{n}^{1} X(\omega)$ 's are not all equal to zero, and recognising that $\sum_{i=0}^{\tau-1} \delta_{\tau n+i}=\sum_{i=0}^{\tau-1} \Delta_{\tau n+i}^{1} X(\omega)=\Delta_{\tau n}^{\tau} X(\omega)$.

In order to prove $(\mathrm{B} .3)$, we take advantage of the fact that $u_{n}^{1 / q}$ and $v_{n}^{1 / q}$ are the first and the second largest of $\left|\delta_{\tau n}\right|,\left|\delta_{\tau n+1}\right|, \ldots,\left|\delta_{\tau n+\tau-1}\right|$ to obtain the straight bounds

$$
\max \left\{0, u_{n}^{\frac{1}{q}}-(\tau-1) v_{n}^{\frac{1}{q}}\right\} \leq\left|\sum_{i=0}^{\tau-1} \delta_{\tau n+i}\right| \leq u_{n}^{\frac{1}{q}}+(\tau-1) v_{n}^{\frac{1}{q}} .
$$

Then, as $0 \leq v_{n} \leq u_{n}$, we invoke $(\underline{\mathrm{B} .1})$ and $(\underline{\mathrm{B} .2})$ with $u:=u_{n}$ and $v:=v_{n}$ to state that for each $n<N$ on the one hand

$$
\left|\sum_{i=0}^{\tau-1} \delta_{\tau n+i}\right|^{q} \geq \begin{cases}u_{n}+(\tau-1) v_{n}-h v_{n} & \text { if } q \leq 1 \\ u_{n}+(\tau-1) v_{n}-h u_{n}^{1-\frac{1}{q}} v_{n}^{\frac{1}{q}} & \text { if } q>1\end{cases}
$$

and on the other hand

$$
\left|\sum_{i=0}^{\tau-1} \delta_{\tau n+i}\right|^{q} \leq \begin{cases}u_{n}+h v_{n} & \text { if } q \leq 1 \\ u_{n}+h u_{n}^{1-\frac{1}{q}} v_{n}^{\frac{1}{q}} & \text { if } q>1\end{cases}
$$


At this point, carrying out the sum over $n$ is what remains to be done. In this respect, we observe that $1-1 / q>0$ when $q>1$ so that the Hölder's inequality applies ensuring that

$$
\sum_{n=0}^{N-1} u_{n}^{1-\frac{1}{q}} v_{n}^{\frac{1}{q}} \leq\left(\sum_{n=0}^{N-1} u_{n}\right)^{1-\frac{1}{q}}\left(\sum_{n=0}^{N-1} v_{n}\right)^{\frac{1}{q}}=\sum_{n=0}^{N-1} u_{n}\left(\frac{\sum_{n=0}^{N-1} v_{n}}{\sum_{n=0}^{N-1} u_{n}}\right)^{\frac{1}{q}}
$$

We notice that $\sum_{n=0}^{N-1} u_{n}$ is different from zero due to the hypothesis that the $\delta_{n}$ 's are not all equal to zero. Carrying out the sum over $n$ in (B.4) and (B.5) and setting $S_{N}:=\sum_{n=0}^{N-1}\left[u_{n}+(\tau-1) v_{n}\right]$ and $T_{N}:=\sum_{n=0}^{N-1} u_{n}$ we reach the result

$$
S_{N}-h T_{N}\left(\frac{\sum_{n=0}^{N-1} v_{n}}{\sum_{n=0}^{N-1} u_{n}}\right)^{e} \leq \sum_{n=0}^{N-1}\left|\sum_{i=0}^{\tau-1} \delta_{\tau n+i}\right|^{q} \leq T_{N}\left[1+h\left(\frac{\sum_{n=0}^{N-1} v_{n}}{\sum_{n=0}^{N-1} u_{n}}\right)^{e}\right],
$$

where $e:=\min \{1,1 / q\}$. This result proves $(\mathrm{B} .3)$ once combined with the fact that

$$
T_{N} \leq \sum_{n=0}^{N-1} \sum_{i=0}^{\tau-1}\left|\delta_{\tau n+i}\right|^{q} \leq S_{N}
$$

as $u_{n} \leq \sum_{i=0}^{\tau-1}\left|\delta_{\tau n+i}\right|^{q} \leq u_{n}+(\tau-1) v_{n}$ for all $n$ by the definition of $u_{n}$ and $v_{n}$.

We conclude the proof of the lemma showing the validity of (B.1) and (B.2). The case $u=0$ is trivial since $v=0$ as $0 \leq v \leq u$. The case $u>0$ descends from the inequalities

$$
1-\xi^{q} \leq(\max \{0,1-\xi\})^{q} \leq(1+\xi)^{q} \leq 1+\xi^{q}
$$

if $q \leq 1$ and

$$
1-\xi^{q}-q \xi \leq(\max \{0,1-\xi\})^{q} \leq(1+\xi)^{q} \leq 1+q(1+\xi)^{q-1} \xi
$$

when $q>1$, which hold for each positive number $\xi$. Verifying such inequalities is a simple exercise of calculus, so we omit the details and move on. The instance $q \leq 1$ of (B.1) and (B.2) follows from (B.6), which after setting $\xi:=(\tau-1)(v / u)^{1 / q}$ and multiplying by $u$ yields

$$
\begin{aligned}
{\left[\max \left\{0, u^{\frac{1}{q}}-(\tau-1) v^{\frac{1}{q}}\right\}\right]^{q} } & \geq u-(\tau-1)^{q} v \\
& =u+(\tau-1) v-\left[\tau-1+(\tau-1)^{q}\right] v \\
& \geq u+(\tau-1) v-h v
\end{aligned}
$$

and

$$
\left[u^{\frac{1}{q}}+(\tau-1) v^{\frac{1}{q}}\right]^{q} \leq u+(\tau-1)^{q} v \leq u+h v
$$

since both $\tau-1+(\tau-1)^{q} \leq 2 \tau$ and $(\tau-1)^{q} \leq 2 \tau$ as $q \leq 1$. The instance $q>1$ of (B.1) and (B.2) follows from (B.7) and the hypothesis $v \leq u$, which in particular entails that $v / u \leq(v / u)^{1 / q}$ and $v \leq u^{1-1 / q} v^{1 / q}$ as a consequence since $v / u \leq 1$ and $1 / q<1$. Setting $\xi:=(\tau-1)(v / u)^{1 / q}$ in (B.7), multiplying by $u$, and bearing in mind that $v \leq u^{1-1 / q} v^{1 / q}$ 
and that $v / u \leq 1$ we find that

$$
\begin{aligned}
{\left[\max \left\{0, u^{\frac{1}{q}}-(\tau-1) v^{\frac{1}{q}}\right\}\right]^{q} } & \geq u-(\tau-1)^{q} v-q(\tau-1) u^{1-\frac{1}{q}} v^{\frac{1}{q}} \\
& =u+(\tau-1) v-\left[\tau-1+(\tau-1)^{q}\right] v-q(\tau-1) u^{1-\frac{1}{q}} v^{\frac{1}{q}} \\
& \geq u+(\tau-1) v-\left[(q+1)(\tau-1)+(\tau-1)^{q}\right] u^{1-\frac{1}{q}} v^{\frac{1}{q}} \\
& \geq u+(\tau-1) v-h u^{1-\frac{1}{q}} v^{\frac{1}{q}}
\end{aligned}
$$

and

$$
\begin{aligned}
{\left[u^{\frac{1}{q}}+(\tau-1) v^{\frac{1}{q}}\right]^{q} } & \leq u+q(\tau-1)\left[1+(\tau-1)\left(\frac{v}{u}\right)^{\frac{1}{q}}\right]^{q-1} u^{1-\frac{1}{q}} v^{\frac{1}{q}} \\
& \leq u+q(\tau-1) \tau^{q-1} u^{1-\frac{1}{q}} v^{\frac{1}{q}} \leq u+h u^{1-\frac{1}{q}} v^{\frac{1}{q}}
\end{aligned}
$$

since both $(q+1)(\tau-1)+(\tau-1)^{q} \leq(q+2) \tau^{q}$ and $q(\tau-1) \tau^{q-1} \leq(q+2) \tau^{q}$ as $q>1$.

\section{Appendix C. Proof of Lemma 3}

Given two numbers $\lambda>0$ and $\xi>0$ the Markov's inequality yields

$$
\begin{aligned}
\mathbb{P}\left[\sum_{n=0}^{N-1} U_{n} \leq \lambda\right] & =\mathbb{P}\left[\exp \left(-\xi \sum_{n=0}^{N-1} U_{n}\right) \geq \exp (-\xi \lambda)\right] \\
& \leq \mathrm{e}^{\xi \lambda} \cdot \mathbb{E}\left[\exp \left(-\xi \sum_{n=0}^{N-1} U_{n}\right)\right]=\mathrm{e}^{\xi \lambda} \cdot \mathbb{E}^{N}\left[\exp \left(-\xi U_{0}\right)\right]
\end{aligned}
$$

where the fact that the $U_{n}$ 's are i.i.d. variables has been used to obtain the last equality. The proof of the lemma moves from this inequality and is based on the property of $U_{0}$ that there exist two constants $\delta>0$ and $\eta>0$, in general depending on both $\beta$ and $\tau$, such that the bound

$$
\mathbb{E}\left[\exp \left(-\xi U_{0}\right)\right] \leq \begin{cases}\mathrm{e}^{-\delta \xi^{\beta}} & \text { if } \beta<1 ; \\ \mathrm{e}^{\delta \xi \ln \xi} & \text { if } \beta=1\end{cases}
$$

holds for positive $\xi \leq \eta$. This bound follows from (12) and will be proven later. Now we observe that combining the Markov's inequality with such bound we get that if $0<\xi \leq \eta$, then

$$
\mathbb{P}\left[\sum_{n=0}^{N-1} U_{n} \leq \lambda\right] \leq \begin{cases}\mathrm{e}^{\xi \lambda-\delta N \xi^{\beta}} & \text { if } \beta<1 \\ \mathrm{e}^{\xi \lambda+\delta N \xi \ln \xi} & \text { if } \beta=1\end{cases}
$$

The lemma is a straight consequence of (C.2) if the right choice of $\xi$ and $\lambda$ is made. For each $N \geq 1$ we set

$$
\xi_{N}:= \begin{cases}{\left[\frac{2}{\delta(1-\beta)}\right]^{\frac{1}{\beta}} N^{-\frac{1}{\beta}}(\ln N+1)^{\frac{1}{\beta}}} & \text { if } \beta<1 \\ \left(\mathrm{e} N^{2}\right)^{-\frac{1}{3}} & \text { if } \beta=1\end{cases}
$$


and we notice that since $\lim _{N \uparrow \infty} \xi_{N}=0$ we can find $N_{0} \geq 1$ with the property that $\xi_{N} \leq \eta$ and $\delta(N / \mathrm{e})^{\frac{1}{3}} \geq 6$ for all $N \geq N_{0}$. Then, taking $\lambda_{N}$ as in the statement of the lemma with

$$
k:= \begin{cases}\beta \delta^{\frac{1}{\beta}}\left(\frac{1-\beta}{2}\right)^{\frac{1-\beta}{\beta}} & \text { if } \beta<1 \\ \frac{\delta}{3} & \text { if } \beta=1\end{cases}
$$

simple algebra shows that $\xi_{N} \lambda_{N}-\delta N \xi_{N}^{\beta}=-2(\ln N+1) \leq-2 \ln N$ when $\beta<1$ and that $\xi_{N} \lambda_{N}+\delta N \xi_{N} \ln \xi_{N}=-(1 / 3) \delta(N / \mathrm{e})^{\frac{1}{3}} \ln N \leq-2 \ln N$ if $\beta=1$ and $N \geq N_{0}$. Thus, plugging $\lambda:=\lambda_{N}$ and $\xi:=\xi_{N}$ in (C.2) we reach the result

$$
\mathbb{P}\left[\sum_{n=1}^{N} U_{n} \leq \lambda_{N}\right] \leq \frac{1}{N^{2}}
$$

for all $N \geq N_{0}$ and the lemma is proven with $K:=N_{0}^{2}$.

To conclude, we show the validity of the bound (C.1). To begin with, given $\xi>0$, we apply the Fubini-Tonelli Theorem to the integral

$$
\int_{0}^{\infty} d x \int_{\Omega} \mathbb{P}[d \omega] \exp (-\xi x) \mathbb{1}\left(U_{0}(\omega)>x\right)
$$

in order to obtain that

$$
\mathbb{E}\left[\exp \left(-\xi U_{0}\right)\right]=1-\xi \int_{0}^{\infty} d x \exp (-\xi x) \mathbb{P}\left[U_{0}>x\right]
$$

Then, as (12) entails that $x^{\beta} \cdot \mathbb{P}\left[U_{0}>x\right] \geq(1 / 2) \tau c$ for all $x \geq s$ with some $s \geq 0$, in general depending on both $\beta$ and $\tau$, we find that

$$
\begin{aligned}
\mathbb{E}\left[\exp \left(-\xi U_{0}\right)\right] & \leq 1-\xi \int_{s}^{\infty} d x \exp (-\xi x) \mathbb{P}\left[U_{0}>x\right] \\
& \leq 1-\frac{\tau c \xi^{\beta}}{2} \int_{\xi s}^{\infty} y^{-\beta} \mathrm{e}^{-y} d y
\end{aligned}
$$

where a change of variable has been performed. On the other hand, there exists a positive number $\eta$, in general depending on both $\beta$ and $\tau$, with the property that for $\xi \leq \eta$ one has $\int_{\xi s}^{\infty} y^{-\beta} \mathrm{e}^{-y} d y \geq \Gamma(1-\beta) / 2$ if $\beta<1, \Gamma$ being the Euler's gamma function, and $\int_{\xi s}^{\infty} y^{-\beta} \mathrm{e}^{-y} d y \geq-\ln \xi / 2$ when $\beta=1$ since $\lim _{\xi \downarrow 0} \int_{\xi s}^{\infty} y^{-\beta} \mathrm{e}^{-y} d y=\Gamma(1-\beta)$ if $\beta<1$ and $\lim _{\xi \downarrow 0}-(1 / \ln \xi) \int_{\xi s}^{\infty} y^{-1} \mathrm{e}^{-y} d y=1$. Thus, combining (C.3) with these bounds we find that for each positive $\xi \leq \eta$

$$
\mathbb{E}\left[\exp \left(-\xi U_{0}\right)\right] \leq \begin{cases}1-\delta \xi^{\beta} & \text { if } \beta<1 \\ 1+\delta \xi \ln \xi & \text { if } \beta=1,\end{cases}
$$

where $\delta:=\tau c \Gamma(1-\beta) / 4>0$ if $\beta<1$ and $\delta:=\tau c / 4>0$ when $\beta=1$. This result gives (C.1) as $1+\zeta \leq \mathrm{e}^{\zeta}$ for any $\zeta$. 


\section{References}

[1] Benzi R, Paladin G, Parisi G, and Vulpiani A, On the multifractal nature of fully developed turbulence and chaotic systems, 1984 J. Phys. A: Math. Gen. 17 3521-3531

[2] Harte J, Kinzig A, and Green J, Self-similarity in the distribution and abundance of species, 1999 Science $284334-336$

[3] Gupta V K and Waymire E C, A statistical analysis of mesoscale rainfall as a random cascade, 1993 J. Appl. Meteor. 32 251-267

[4] Leland W, Taqqu M, Willinger W, and Wilson D, On the self-similar nature of ethernet traffic, 1994 IEEE/ACM Trans. Netwo. 2 1-15

[5] Di Matteo T, Multi-scaling in finance, 2007 Quant. Finance 7 21-36

[6] Mandelbrot B B, Fisher A, and Calvet L, A multifractal model of asset returns. Cowles Foundation Discussion Papers No. 1164, 1997 Yale University

[7] Calvet L and Fisher A, Multifractality in asset returns: theory and evidence, 2002 Rev. Econ. Stat. $84381-406$

[8] Schmitt F, Schertzer D, and Lovejoy S, Multifractal analysis of foreign exchange data, 1999 Appl. Stoch. Models Data Anal. 15 29-53

[9] Xu Z and Gençay R, Scaling, self-similarity and multifractality in FX markets, 2003 Physica A $323578-590$

[10] Wei Y and Huang D, Multifractal analysis of SSEC in Chinese stock market: a different empirical result from Heng Seng index, 2005 Physica A 355 497-508

[11] Jiang Z Q and Zhou W X, Multifractal analysis of Chinese stock volatilities based on the partition function approach, 2008 Physica A 387 4881-4888

[12] Zunino L, Figliola A, Tabak B M, Pérez D G, Garavaglia M, and Rosso O A, Multifractal structure in Latin-American market indices 2009 Chaos Solitons Fractals 41 2331-2340

[13] Green E, Hanan W, and Heffernan D, The origins of multifractality in financial time series and the effect of extreme events, 2014 Eur. Phys. J. B 87129

[14] Jiang Z Q and Zhou W X, Multifractality in stock indexes: fact or fiction?, 2008 Physica A 387 3605-3614

[15] Zhou W X, The components of empirical multifractality in financial returns 2009 Europhys. Lett. 8828004

[16] Zhou W X, Finite-size effect and the components of multifractality in financial volatility 2012 Chaos Solitons Fractals 45 147-155

[17] Chechkin A and Gonchar V Y, Self and spurious multi-affinity of ordinary Lévy motion, and pseudo-Gaussian relations, 2000 Chaos Solitons Fractals 11 2379-2390

[18] Bouchaud J P, Potters M, and Meyer M, Apparent multifractality in financial time series, 2000 Eur. Phys. J. B 13 595-599

[19] Kiyani K, Chapman S C, and Hnat B, Extracting the scaling exponents of a self-affine, nonGaussian process from a finite-length time series, 2006 Phys. Rev. E 74051122

[20] Heyde C C and Sly A, A cautionary note on modeling with fractional Lévy flights, 2008 Physica A 387 5024-5032

[21] Heyde C C, Scaling issues for risky asset modelling, 2009 Math. Method. Oper. Res. 69 593-603

[22] Neuman S P, Apparent/spurious multifractality of data sampled from fractional Brownian/Lévy motions, 2010 Hydrol. Process. 24 2056-2067

[23] Neuman S P, Apparent/spurious multifractality of absolute increments sampled from truncated fractional Gaussian/Lévy noise, 2010 Geophys. Res. Lett. 37 L09403

[24] Guadagnini A, Neuman S P, and Riva M, Numerical investigation of apparent multifractality of samples from processes subordinated to truncated fBm, 2012 Hydrol. Process. 26 2894-2908

[25] Grahovac D and Leonenko N N, Detecting multifractal stochastic processes under heavy-tailed effects, 2014 Chaos Solitons Fractals 65 78-89

[26] Cont R, Empirical properties of asset returns: stylized facts and statistical issues, 2001 Quant. 
Finance $1223-236$

[27] Matsushita R, Gleria I, Figueiredo A, Rathie P, and Da Silva S, Exponentially damped Lévy flights, multiscaling, and exchange rates, 2004 Physica A 333 353-369

[28] Watkins N W, Credgington D, Hnat B, Chapman S C, Freeman M P, and Greenhough J, Towards synthesis of solar wind and geomagnetic scaling exponents: a fractional Lévy motion model, 2005 Space Sci. Rev. 121 271-284

[29] Gal N and Weihs D, Experimental evidence of strong anomalous diffusion in living cells, 2010 Phys. Rev. E 81020903

[30] Dechant A and Lutz E, Anomalous spatial diffusion and multifractality in optical lattices, 2012 Phys. Rev. Lett. 108230601

[31] Muzy J F and Bacry E, Multifractal stationary random measures and multifractal random walks with log infinitely divisible scaling laws, 2002 Phys. Rev. E 66056121

[32] Bacry E and Muzy J F, Log-infinitely divisible multifractal processes, 2003 Commun. Math. Phys. $236449-475$

[33] Sato K I, 1999 Lévy processes and infinitely divisible distributions. Vol. 68 of Cambridge Studies in Advanced Mathematics. Cambridge: Cambridge University Press

[34] Zolotarev V M, 1986 One-dimensional stable distributions. In: Translations of Mathematical Monographs, Vol. 65, Providence, RI: Amer. Math. Soc., Translated from the Russian by H. H. McFaden, Translation edited by Ben Silver

[35] Feller W, 1971 An introduction to probability theory and its application. Vol. 2. Second edition, New York: John Wiley \& Sons Inc.

[36] Durrett R, 2010 Probability: Theory and Examples. Fourth edition, Cambridge: Cambridge University Press 